\title{
Novel Applicators for Local Microwave Hyperthermia Based on Zeroth-Order Mode Resonator Metamaterial
}

\author{
David Vrba and Jan Vrba \\ Department of Biomedical Technology, Faculty of Biomedical Engineering, Czech Technical University in Prague, \\ Zikova 4, 16636 Prague, Czech Republic
}

Correspondence should be addressed to David Vrba; david.vrba@fbmi.cvut.cz

Received 17 January 2014; Accepted 11 March 2014; Published 9 April 2014

Academic Editor: Haider Raad Khaleel

Copyright (C) 2014 D. Vrba and J. Vrba. This is an open access article distributed under the Creative Commons Attribution License, which permits unrestricted use, distribution, and reproduction in any medium, provided the original work is properly cited.

\begin{abstract}
It is demonstrated that a theory of zero-order mode resonator (ZOR) metamaterial (MTM) structure can be used for the development of a novel class of applicators for microwave thermotherapy, for example, for hyperthermia in cancer treatment or for physiotherapy. The main idea of creating such an applicator is to generate and radiate a plane electromagnetic (EM) wave into the treated biological tissue, at least in a certain extent. The main aim of this paper is to investigate whether an EM wave generated by ZOR MTM structure and emitted into the biological tissue can produce a homogeneous SAR distribution in the planes parallel to the applicator aperture and achieve a penetration depth approaching the theoretical limit represented by SAR distribution and penetration depth of an ideal EM plane wave. EM field distribution inside a virtual phantom of the treated region generated by the applicator that is based on the proposed ZOR MTM principle is investigated using a well-proven full-wave commercial simulation tool. The proposed applicator type shows both a low unwanted leaked electromagnetic field and a fairly homogeneous electric field in its aperture as well as in the virtual phantom of the treated region.
\end{abstract}

\section{Introduction}

The main aim of this paper is to verify whether it is feasible to apply principles of ZOR MTM radiating structures in the design of efficient applicators for radiofrequency (RF)/ microwave (MW) thermotherapy, especially for hyperthermia cancer treatment and for physiotherapy. For this aim, MTM antennas inspired by those described in $[1,2]$ will be investigated and adapted with respect to requirements of effective hyperthermia treatment (i.e., we will study the homogeneity of SAR distribution in the treated region). In order to achieve an optimal waveform shape of the radiated EM wave, applicators have to be designed in a certain way. The best possible results can be obtained by comparing various shapes of waveforms of EM waves (e.g., plane wave, cylindrical wave, and spherical wave) for local and deep local treatment waveform of plane wave. This can ensure

(i) the best possible value of the effective treatment depth;

(ii) the best possible homogeneity of SAR 3D distribution (i.e., its distribution on the surface and in the whole volume of the area to be treated).
SAR distribution inside the virtual phantom of the treated region, created using the proposed applicator, and the reflection coefficient of the proposed applicator are investigated using the well-proven full-wave commercial simulation tool COMSOL Multiphysics. Based on our previous experience [1-3], an excellent agreement between simulation and measurement results can be expected. That is why the conclusions made in this paper are based on the results of numerical simulations only.

\section{MTM Zeroth-Order Mode Resonator}

The concept of MTM phenomenon was first comprehensively introduced by Veselago in 1968 [4]. In the aforementioned publication, he speculated on the existence of materials whose permittivity $(\varepsilon)$ and permeability $(\mu)$ were simultaneously negative. He named these materials left-handed (LH), as the $\mathbf{E}, \mathbf{H}$, and $\mathbf{k}$ vectors of the studied EM wave formed a lefthanded triad if the wave propagated through such environment. The first experimental verification of MTM phenomena was performed by a research group at the University of California, San Diego (UCSD), in 2001 [5]. 


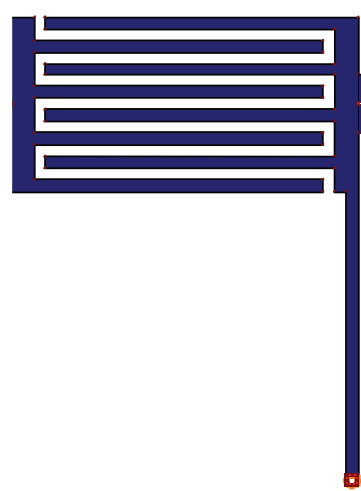

(a)

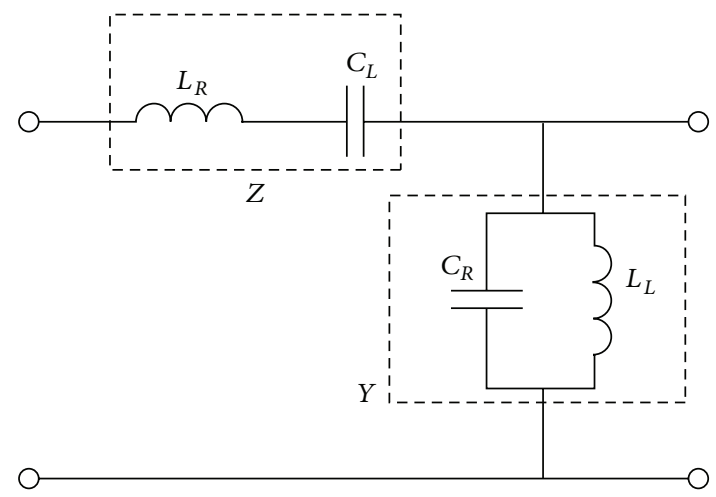

(b)

Figure 1: (a) Infinitesimal element of MTM (b) and its equivalent circuit consisting of inherent series inductor $L_{R}$, shunt capacitor $C_{R}$, artificially inserted series capacitor $C_{L}$, and shunt inductor $L_{L}$.

An infinitesimally short lossless transmission line (TL) section can be described by a simple equivalent circuit consisting of a series inductor $L_{R}$ and a shunt capacitor $C_{R}$ [6]. The lossless MTM cell implemented in planar technology consists of a TL section with artificially inserted series capacitors $C_{L}$ and shunt inductors $L_{L}$ (with subscript $L$ denoting its left-handed properties). The equivalent circuit of the MTM cell can be then represented by four-lumped elements (as shown in Figure 1) [6].

Several different EM radiating structures based on the MTM principle were introduced in the past [7-10]. Since the very beginning of the development, however, real implementation possibilities of such antennas, for example, in communication technology, have been very limited because of their poor radiation efficiency. MTM antennas with very good radiation efficiency were first presented in $[1,2]$.

\section{Design of the Applicator Based on ZOR MTM Structure}

In this section, we will study the possibility of creating MTM based applicators for local microwave hyperthermia cancer treatment at a frequency of $434 \mathrm{MHz}$. The basic part of the proposed applicator will consist of the ZOR.

The working idea of ZOR is based on a special case of resonance that can occur when the TL meets the conditions of the MTM phenomenon. The phase constant $\beta=0$ is at working frequency in this case, which implies infinite guided wavelength $\lambda_{g}=2 \pi /|\beta|$ along the MTM structure as well as zero phase shift $\left(\theta_{m}=-\beta l=0\right)$ [11]. It is very important to note that this phenomenon enables creation of a very special kind of resonator whose physical length is completely independent of the classical resonance condition (i.e., required to be a natural number multiple of the half working wavelength in case of either open-circuited or short-circuited TL) [11]. The typical voltage wave distribution along the resonant length for the negative $(m<0)$ and zero $(m=0)$ resonances is shown in Figure 2. In the case of zeroth-order mode resonance $(m=0)$, the value of the voltage along the

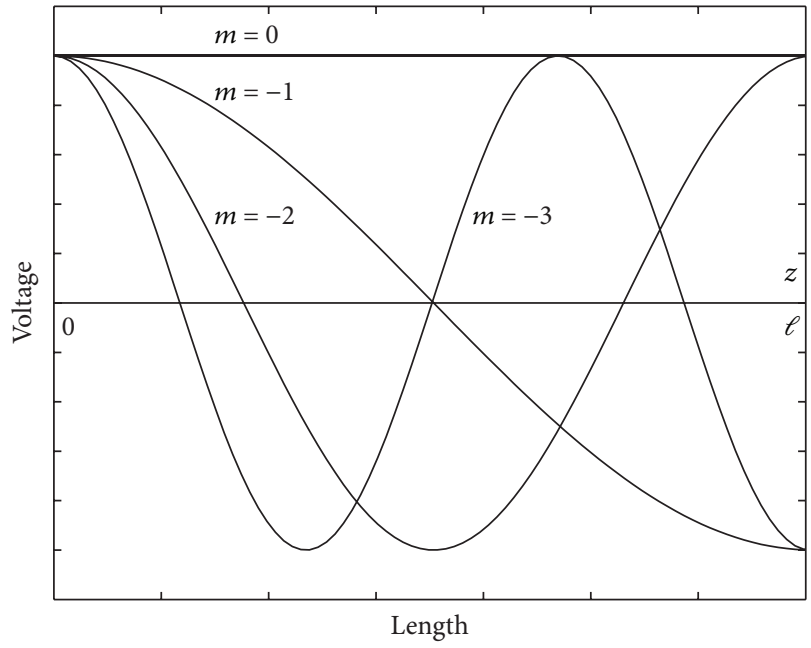

FIGURE 2: Voltage distribution in case of open-circuited TL of length $\ell$. Mode $m=0$ represents the ZOR with infinite guided wavelength.

$\mathrm{ZOR}$ is constant. A more comprehensive description of ZOR properties can be found in [11].

The zeroth-order resonant frequency of the proposed ZOR MTM applicator is equal to the working frequency of a particular RF/MW hyperthermia system. Please note that the ideas and principles of MTM applicator design described here are expected to be valid at any usual working frequency of RF/MW hyperthermia systems. The initial idea of the mechanical and EM arrangements of the ZOR MTM applicator proposed here is displayed in Figure 3. In order to obtain high radiation efficiency and thus the best possible transfer of EM energy to the treated area, we can use the main ideas, experiences, contributions, and conclusions described in [1-3]. That means to combine the ZOR MTM structure with relatively long inductive elements along which electric current with equal phase will flow. These currents will then excite an EM wave propagating into the area to be treated. Optimal choice of positions of these inductive elements then enables us to approximate the preferred waveform (we want to excite 


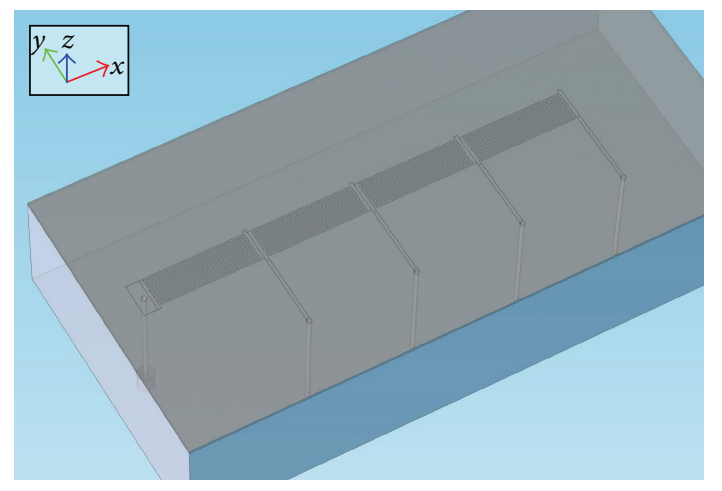

FIGURE 3: Example of the ZOR MTM applicator consisting of a fourunit cell.

the waveform of a plane wave, but the waveform of either a cylindrical or a spherical wave can be obtained as well). This will enable us to approximate the possible shape as well as the dimensions of the treated area. For the above mentioned main aim, some results and experiences obtained by applicators described in [12-16] can be used.

The design rules of this structure are the same as in [1]. Thanks to the excitation of zeroth-order mode vectors of surface current density in all vertical parts of the antenna (including feeding), all these surface currents are in phase; that is, the radiated contributions from all individual vertical parts are in a very good superposition in the applicator aperture (i.e., the Huygens principle phenomenon can be applied to describe the resulting EM field distribution in the area to be treated). For the design of the ZOR MTM applicators, the following dimensions of antenna elements were chosen. In this special discussed case, the length of the vertical part of the antenna is equal to $\lambda_{0} / 10$. The longitudinal dimension of the unit cell can vary (or be adjusted) in a relatively large range [17]. Depending on the selected size of this dimension, it is necessary to adjust the dimensions of interdigital capacitors. For the presented applicator, the overall length of one unit cell is $p=70 \mathrm{~mm}$, the length of figures of the interdigital capacitor equals $l_{\text {capacitor }}=68 \mathrm{~mm}$, and the width and the gap of the interdigital capacitor are $w=s=1 \mathrm{~mm}$; please note that the same notation has been used as in [2]. The overall physical dimensions of the applicator prototype are $268 \times 68 \times$ $70 \mathrm{~mm}$ which represents relative dimensions (with respect to wavelength of plane EM wave in vacuum $\lambda_{0}$ at working frequency) as follows: the relative width of the aperture is equal to $\lambda_{0} / 3$, the relative height of the aperture is $\lambda_{0} / 10$, and the relative depth of the applicator is $\lambda_{0} / 10$. Thickness, relative permittivity, and equivalent conductivity of the considered substrate are $1.5 \mathrm{~mm}, 4.3$, and $0.001 \mathrm{~S} / \mathrm{m}$, respectively. Alternatively, the ZOR MTM radiating structure can be inserted into the rectangular waveguide section to ensure that the whole radiated power would be perfectly directed to the biological tissue in the area to be treated. Another possible and interesting use for real clinical therapy is the case where the ZOR MTM radiating structure would be surrounded by metal plates from the top and the back sides and the lateral sides would be made of a dielectric substrate material to

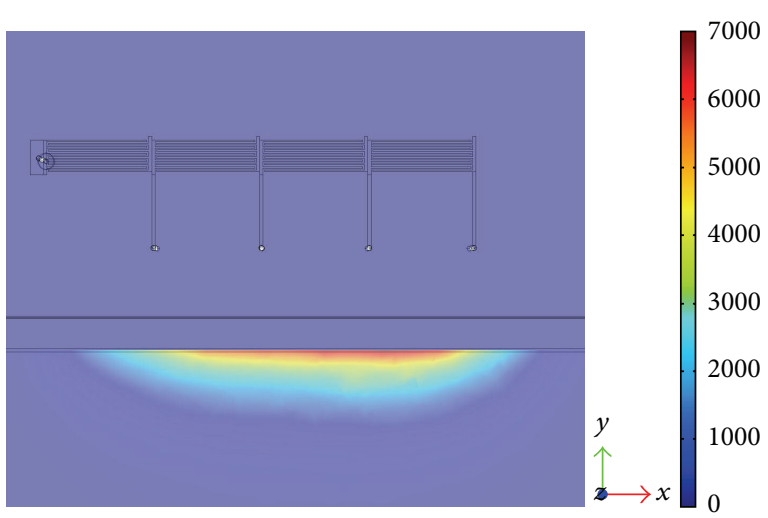

FIgURE 4: Top view of the applicator, water bolus, and the tissue to be treated. SAR distribution in the area to be treated is also displayed here.

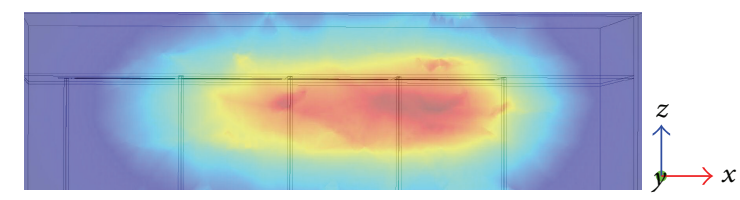

FIGURE 5: SAR distribution calculated on the surface of the area to be treated. The color bar here is the same as in Figure 4.

ensure that we would not excite the dominant rectangular waveguide mode $T E_{10}$. One of the basic features of the ZOR MTM radiating structure, observed during our investigations, is that it resonates at almost the same frequency irrespective of whether the structure is surrounded by metal from the back and lateral sides. Only if we placed the metal plane closer to the top of ZOR, the capacity of interdigital capacitors would change and thus the resonant frequency could be shifted from the working frequency. This phenomenon can be used for frequency adjusting or impedance matching of this applicator if necessary.

\section{Distribution of SAR Created by ZOR MTM in the Treated Area}

To study and verify how these ZOR MTM structures would radiate into the biological tissue in the area to be treated, several numerical simulations of the discussed case were performed. SAR distribution inside a virtual phantom of the treated region generated by the proposed applicator was investigated using COMSOL Multiphysics [18]. Muscle tissue dielectric parameters were considered as follows: the real part of the complex permittivity is equal to $\varepsilon_{r}=57$ and the equivalent electric conductivity is equal to $\sigma_{e}=0.81 \mathrm{~S} / \mathrm{m}$ [19]. In the studied model displayed in Figure 4, a water bolus with a thickness of $2 \mathrm{~cm}$ was placed between the applicator aperture and the virtual phantom in the area to be treated. The resulting SAR distribution is displayed in two planes in Figures 4 and 5. Figure 4 displays SAR distribution in the plane perpendicular to the applicator aperture, illustrating how deep EM power penetrates into the area to be treated. 


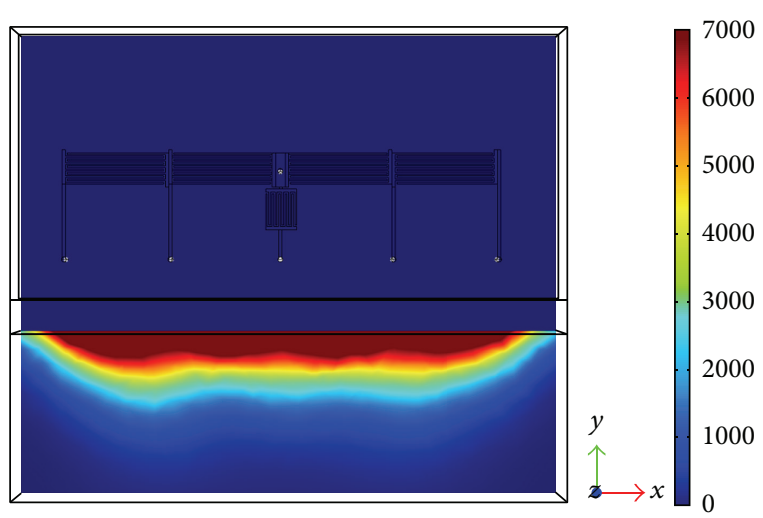

Figure 6: Top view of the applicator with symmetrical feeding, water bolus, and the tissue. SAR distribution in the area to be treated is also displayed here.

It can be observed that when the EM power penetrates the biological tissue, it has very good SAR homogeneity and that the penetration depth is approaching the theoretical limit [20].

Figure 5 displays SAR distribution in the plane parallel to the applicator aperture. Homogeneous absorption of EM at the surface of the area to be treated can be observed. Similar distribution of SAR will be observed in all planes parallel to the applicator aperture, but the level of SAR will decrease with increasing depth.

\section{Improvement of SAR Homogeneity by Aid of Symmetric Feeding of ZOR MTM}

The ZOR MTM applicator discussed in this paper and the distribution of SAR achieved by the use thereof (displayed in Figures 4 and 5) can be considered very suitable for practical treatment of cancer patients. As already mentioned in this paper, it approaches the homogeneity level and the effective treatment depth of the plane wave. However, a certain level of asymmetry of SAR distribution is evident in Figures 4 and 5. This effect can be explained by asymmetrical feeding of the ZOR MTM applicator displayed in Figures 5 and 6 and also by the fact that the surface current density on the feeding vertical part has the same phase as the surface current density on the other vertical parts.

The contribution to radiation is not as it could be if the feeding part would be in the same line as the others. In this part, a modified mechanical and EM arrangement of the ZOR MTM is proposed, which would help us improve the homogeneity of SAR distribution in the treated area. As can be seen in Figure 6, a modified ZOR MTM applicator with symmetric feeding is proposed. Furthermore, another vertical radiating part connected to the feeding point via an interdigital capacitor and a section of microstrip TL that was added to ensure the same phase shift as that of the other vertical parts.

The working principle of this ZOR MTM structure is the same as in the previous case. Thanks to the excitation of the zeroth-order mode, the vectors of surface current density on

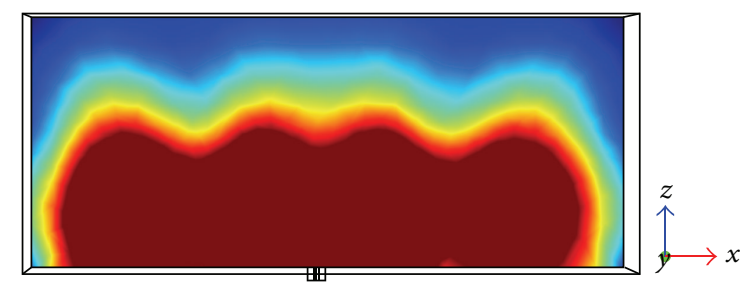

FIGURE 7: SAR distribution in the plane parallel to the symmetrical fed applicator aperture. The color bar here is the same as in Figure 6.

all vertical parts of the antenna including the feeding are in phase. Again, we can say that the phenomenon of the Huygens principle can be applied to describe the resulting EM field distribution in the area to be treated. Therefore, the radiated contributions from all vertical parts constructively interfere in the applicator aperture. The dimensions of the vertical parts of the antenna were chosen to be $\lambda / 10$ again. The longitudinal dimension of the unit cell can be varied (be adjusted) in a relatively large range [17]. Based on the choice of this dimension, it is necessary to adjust the dimensions of interdigital capacitors.

In Figures 6 and 7, it is evident that the homogeneity of SAR in front of five symmetrically fed radiating parts is much better than the one described in the previous case (chapters 3 and 4). Figure 6 displays SAR distribution in the plane perpendicular to the applicator aperture, illustrating how deep EM energy penetrates the area to be treated. It can be observed that when the EM wave penetrates the biological tissue, it has very homogeneous SAR distribution and that both the SAR distribution and the depth of penetration of the treated area approach the theoretical limit of a plane EM wave.

Figure 7 displays SAR distribution in the plane parallel to the applicator aperture, illustrating the homogeneity of EM energy absorption at the surface of the area to be treated. Similar distribution of SAR will be observed in all planes parallel to the applicator aperture, but the level of SAR will decrease with increasing depth.

\section{Achieved Waveform and Comparison with Theoretical Limits}

In this section, the achieved wave form in the biological tissue is compared to the theoretical limit represented by plane EM wave.

SAR distribution in Figure 7 shows very good homogeneity. In Figure 8, SAR distribution along three line segments (cut lines) which lie in the plane perpendicular to the water bolus/treated area interface is depicted. The plane cuts the inductive posts (vertical parts) in the middle. Cut lines 1, 2, and 3 start at the interface, lead into the treated area, and are located between the first and the second inductive posts, between the fourth and the fifth inductive posts, and in front of the third inductive post, respectively. SAR distribution is compared to that of plane EM wave propagating through the treated area considered here (muscle tissue). SAR values are given as percentages of the SAR value of the plane EM 


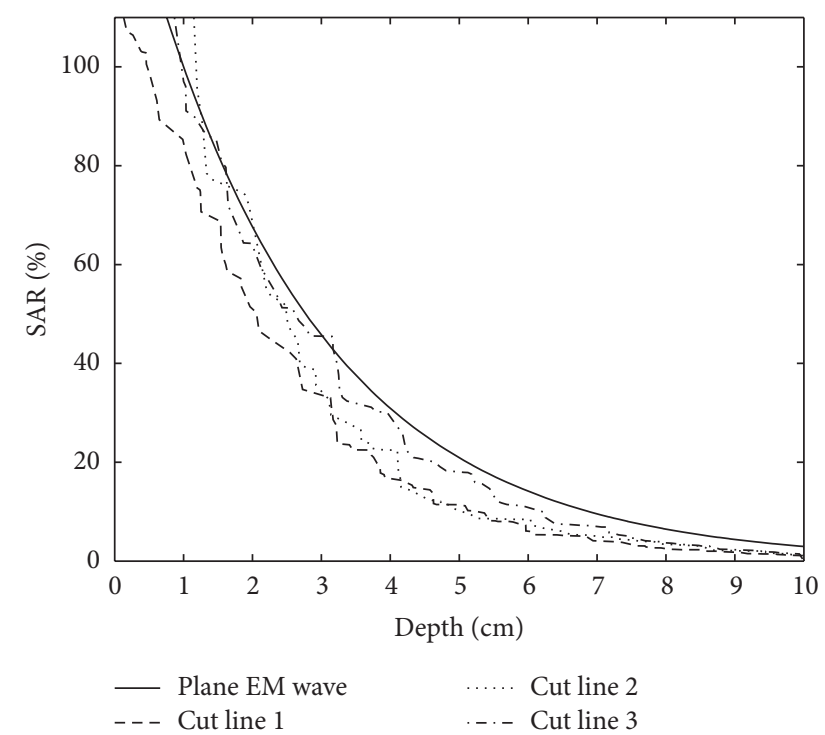

FIGURE 8: SAR distribution corresponding to plane EM wave propagating through the treated area considered here (muscle tissue). SAR distribution along three line segments (cut lines) which lie in the plane perpendicular to the water bolus/treated area interface. The plane cuts the inductive posts in the middle. Cut lines 1,2, and 3 start at the interface, lead into the treated area, and are located between the first and the second inductive posts, between the fourth and the fifth inductive posts, and in front of the third inductive post, respectively. The SAR values are given as percentages of the SAR value of the plane EM wave at the depth of $1 \mathrm{~cm}$.

wave at the depth of $1 \mathrm{~cm}$. Along all three cut lines, the SAR approximates very well the shape of the exponential form related to plane EM wave. In Figure 9, SAR distribution along three line segments (cut lines) which lie in the plane perpendicular to the water bolus/treated area interface is plotted. The plane cuts the inductive posts in the middle. The cut lines are parallel to the interface. Cut lines 4, 5, and 6 are located at the interface, at a depth of 1,2 , and $3 \mathrm{~cm}$, respectively. SAR values are given as percentages and are related to the highest SAR value along cut line 5 . At the depth of $1 \mathrm{~cm}$, SAR distribution is fairly homogeneous. The heat produced by the hotspots at the surface has to be removed using the water bolus, a common strategy used in RF/MW hyperthermia. Similarly, in Figure 10, SAR distribution along three line segments (cut lines) which lie in the plane perpendicular to the water bolus/ treated area interface is plotted. The plane is parallel and passes through the third inductive post. The cut lines are parallel to the interface. Cut lines 7, 8, and 9 are located at the interface, at a depth of 1,2 , and $3 \mathrm{~cm}$, respectively. SAR values are given in percent and are related to the highest SAR value along cut line 8 .

In Figures 11 and 12, contours of SAR values as percentages in two main planes are plotted, in the plane parallel to the surface of the treated biological tissue in the depth of $1 \mathrm{~cm}$ in the tissue and in the plane perpendicular to this plane, cutting the inductive posts in the middle. 25,50 , and $75 \%$ SAR levels are plotted, related to the highest SAR value in the depth of $1 \mathrm{~cm}$ in the tissue. Usually, the 50\% SAR contour is considered

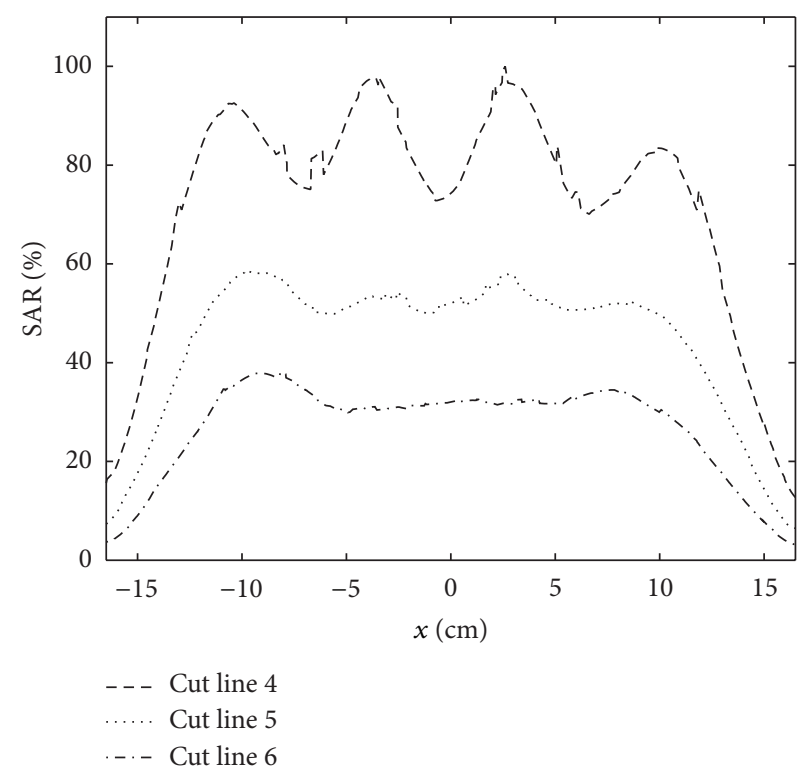

FIGURE 9: SAR distribution along three line segments (cut lines) which lie in the plane perpendicular to the water bolus/treated area interface. The plane cuts the inductive posts in the middle. The cut lines are parallel to the interface. Cut lines 4, 5, and 6 are located at the interface, at a depth of 1,2 , and $3 \mathrm{~cm}$, respectively. SAR values are given as percentages of and are related to the highest SAR value along cut line 4 .

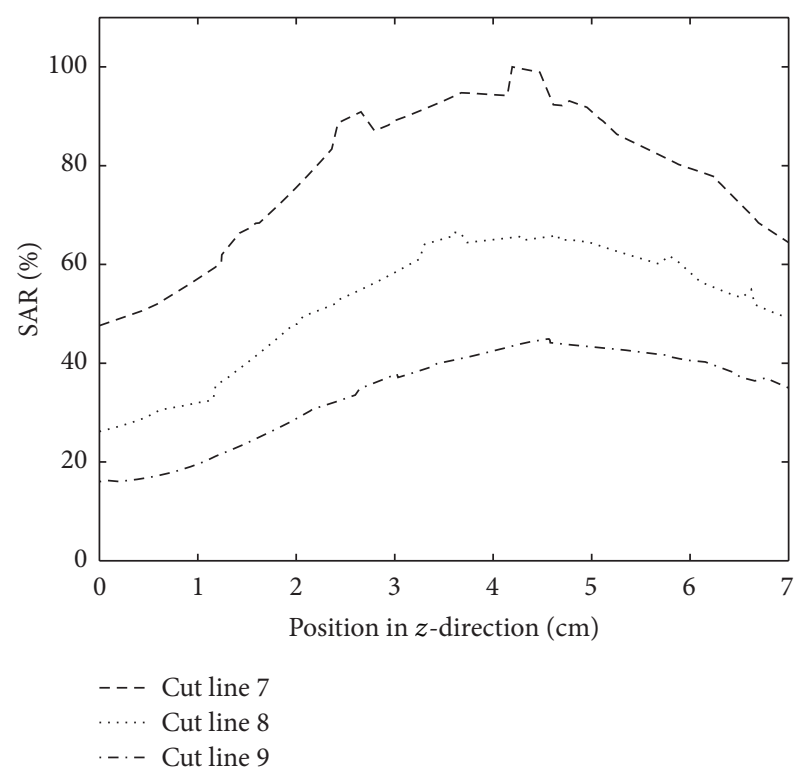

FIGURE 10: SAR distribution along three line segments (cut lines) which lie in the plane perpendicular to the water bolus/treated area interface. The plane is parallel and passes through the third inductive post. The cut lines are parallel to the interface. Cut lines 7, 8, and 9 are located at the interface, at a depth of 1,2 , and $3 \mathrm{~cm}$, respectively. SAR values are given as percentages of the highest SAR value along cut line 7. 


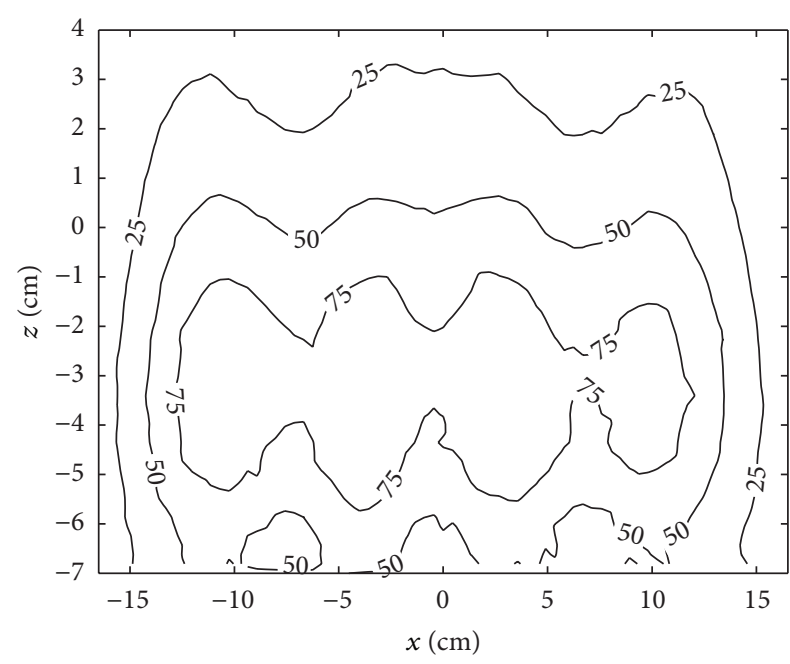

FIGURE 11: Contours of SAR distribution in the plane parallel to the applicator aperture. The plane is located $1 \mathrm{~cm}$ inside the phantom of the treated tissue and SAR values are given as percentages of the highest value in the considered plane.

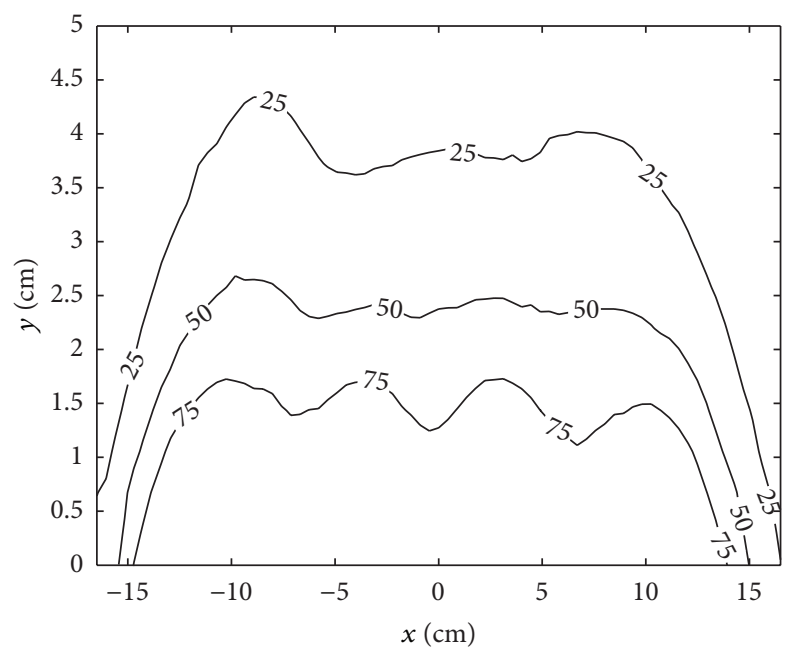

FIGURE 12: Contours of SAR distribution in the plane perpendicular to the applicator aperture. The plane cuts the inductive posts in the middle. SAR values are given as percentages of the highest value in the considered plane at the depth of $1 \mathrm{~cm}$.

to bound the area where the treatment is efficient. From Figure 11, it follows that the surface area of a rectangular effective treatment area (a rectangle inscribed in the $50 \%$ SAR contour) for the proposed applicator is about 6 by $24 \mathrm{~cm}^{2}$.

\section{Ongoing Research of ZOR MTM Applicators}

Our ongoing research of ZOR MTM applicators focuses on designing several practical clinical applicators and on preparing and performing basic experimental evaluations of these structures in order to verify the promising results presented here, obtained by numerical simulations only. We explore two basic structure types:

(i) ZOR MTM structure inserted in the waveguide (thus gaining the advantages of waveguide applicators);

(ii) ZOR MTM structure used as a flat planar applicator.

\section{Conclusions}

In this paper, a novel principle for the design of applicators based on ZOR MTM structures has been proposed. It has been demonstrated here that when penetrating biological tissue EM waves generated by the proposed applicators generate very good SAR homogeneity and achieve penetration depth approaching, to a certain extent, the theoretical limit, that is, close to that of the EM plane wave. The surface of a rectangular effective treatment area (a rectangular inscribed into $50 \%$ SAR contour) is for the proposed applicator about $6 \times$ $24 \mathrm{~cm}^{2}$.

\section{Conflict of Interests}

The authors declare that there is no conflict of interests regarding the publication of this paper.

\section{Acknowledgments}

This research has been supported by the research program of the Grant Agency of the Czech Republic, Project no. 1329857P Human Body Interactions with EM Field Radiated by Metamaterial Structures and Project no. 14-00386P Study of Thermal and Nonthermal Effects of High-Power EM Field Structure of Matter.

\section{References}

[1] D. Vrba and M. Polívka, "Radiation efficiency improvement of zeroth-order resonator antenna," Radioengineering, vol. 18, no. 1, pp. 1-8, 2009.

[2] M. Polívka and D. Vrba, "Shielded micro-coplanar CRLH TL zeroth-order resonator antenna: critical performance evaluation," Radioengineering, vol. 18, no. 4, pp. 368-372, 2009.

[3] D. Vrba, Electrically miniaturized antennas based on Zerothorder resonance [Ph.D. thesis], Czech Technical University in Prague, Faculty of Electrical Engineering, Prague, Czech Republic, 2012.

[4] V. Veselago, "The electrodynamics of substances with simultaneously negative values of permittivity and permeability?" Soviet Physics Uspekhi, vol. 10, no. 4, pp. 509-514, 1968.

[5] R. A. Shelby, D. R. Smith, and S. Schultz, "Experimental verification of a negative index of refraction," Science, vol. 292, no. 5514, pp. 77-79, 2001.

[6] D. M. Pozar, Microwave Engineering, John Wiley and Sons, New York, NY, USA, 2nd edition, 1998.

[7] F. Qureshi, M. A. Antoniades, and G. V. Eleftheriades, "A compact and low-profile metamaterial ring antenna with vertical polarization," IEEE Antennas and Wireless Propagation Letters, vol. 4, no. 1, pp. 333-336, 2005. 
[8] J.-H. Park, Y.-H. Ryu, J.-G. Lee, and J.-H. Lee, "A zeroth-order resonator antenna using epsilon negative meta-structured transmission line," in Proceedings of the IEEE Antennas and Propagation Society International Symposium, pp. 3480-3483, June 2007.

[9] H. R. Raad, A. I. Abbosh, H. M. Al-Rizzo, and D. G. Rucker, "Flexible and compact AMC based antenna for telemedicine applications," IEEE Transactions on Antennas and Propagation, vol. 61, no. 2, pp. 524-531, 2013.

[10] S. Pyo, S. Han, J. Baik, and Y. Kim, "A slot-loaded composite right/left-handed transmission line for a zeroth-order resonant antenna with improved efficiency," IEEE Transactions on Microwave Theory and Techniques, vol. 57, no. 11, pp. 2775-2782, 2009.

[11] C. Caloz and T. Itoh, Electromagnetic Metamaterials: Transmition Line Theory and Microwave Applications, John Wiley and Sons, New York, NY, USA, 2006.

[12] C. Franconi, J. Vrba Jr., and F. Montecchia, “27 MHz hybrid evanescent-mode applicators (HEMA) with flexible heating field for deep and safe subcutaneous hyperthermia," International Journal of Hyperthermia, vol. 9, no. 5, pp. 655-673, 1993.

[13] J. Vrba Jr., C. Franconi, F. Montecchia, and I. Vannucci, "Evanescent-mode applicators (EMA) for superficial and subcutaneous hyperthermia," IEEE Transactions on Biomedical Engineering, vol. 40, no. 5, pp. 397-407, 1993.

[14] P. Togni, Z. Rijnen, W. C. M. Numan et al., "Electromagnetic redesign of the HYPERcollar applicator: toward improved deep local head-and-neck hyperthermia," Physics in Medicine and Biology, vol. 58, pp. 5997-6009, 2013.

[15] H. D. Trefná, J. Vrba, and M. Persson, “Time-reversal focusing in microwave hyperthermia for deep-seated tumors," Physics in Medicine and Biology, vol. 55, no. 8, pp. 2167-2185, 2010.

[16] H. D. Trefná, J. Vrba, and M. Persson, "Evaluation of a patch antenna applicator for time reversal hyperthemia," International Journal of Hyperthermia, vol. 26, no. 2, pp. 185-197, 2010.

[17] M. Polivka and D. Vrba, "Input resistance of electrically short not-too-closely spaced multi- element monopoles with uniform current distribution," IEEE Antennas and Wireless Propagation Letters, vol. 11, no. 1, pp. 1592-1595, 2012.

[18] COMSOL AB, COMSOL Multiphysics User's Guide, COMSOL AB, Stockholm, Sweden, 4th edition, 2010.

[19] D. Andreuccetti, R. Fossi, and C. Petrucci, "An internet resource for the calculation of the dielectric properties of body tissues in the frequency range $10 \mathrm{~Hz}-100 \mathrm{GHz}$," March 2013.

[20] J. Vrba, C. Franconi, and M. Lapes, "Theoretical limits for the penetration depth of intracavitary applicators," International Journal of Hyperthermia, vol. 12, no. 6, pp. 737-742, 1996. 

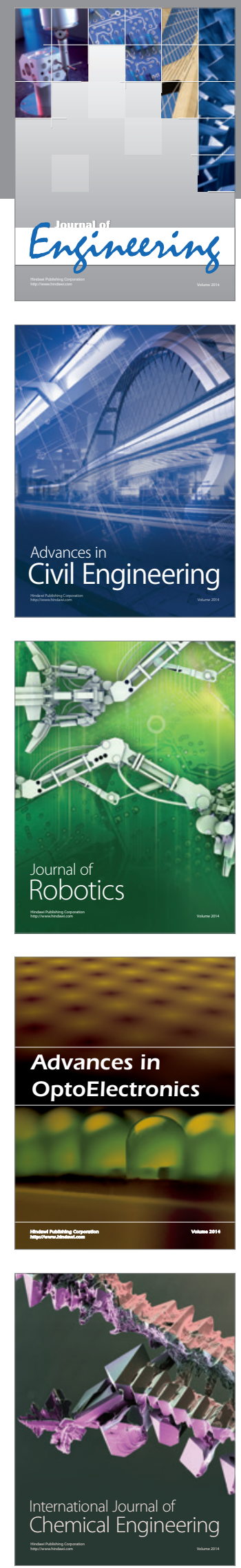

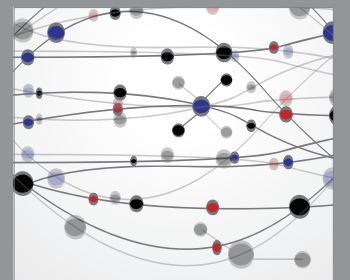

The Scientific World Journal
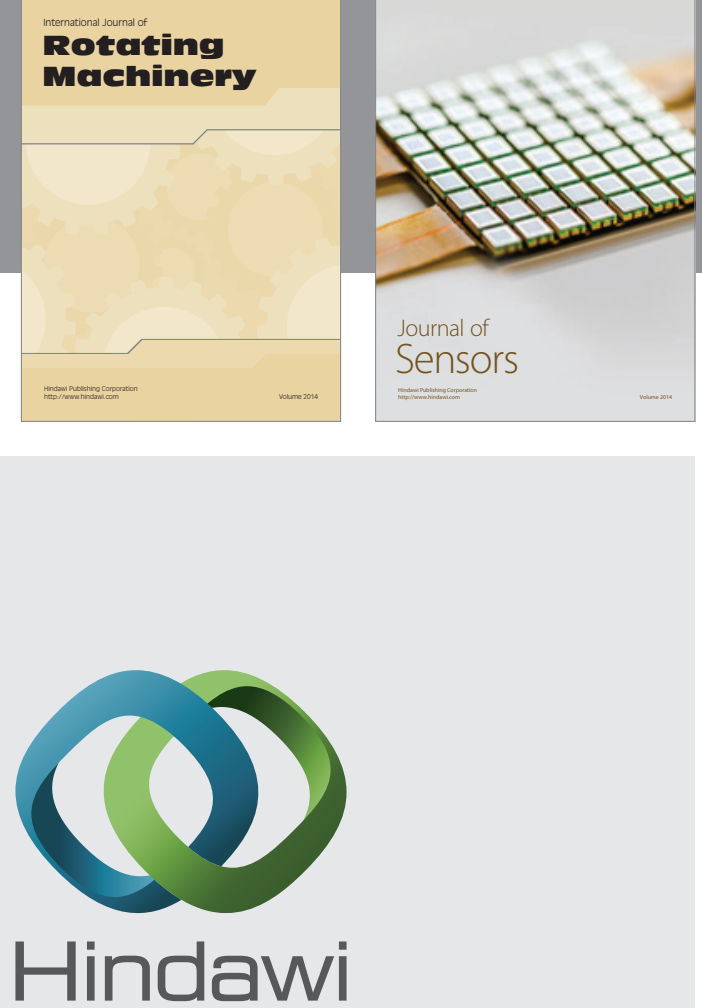

Submit your manuscripts at http://www.hindawi.com
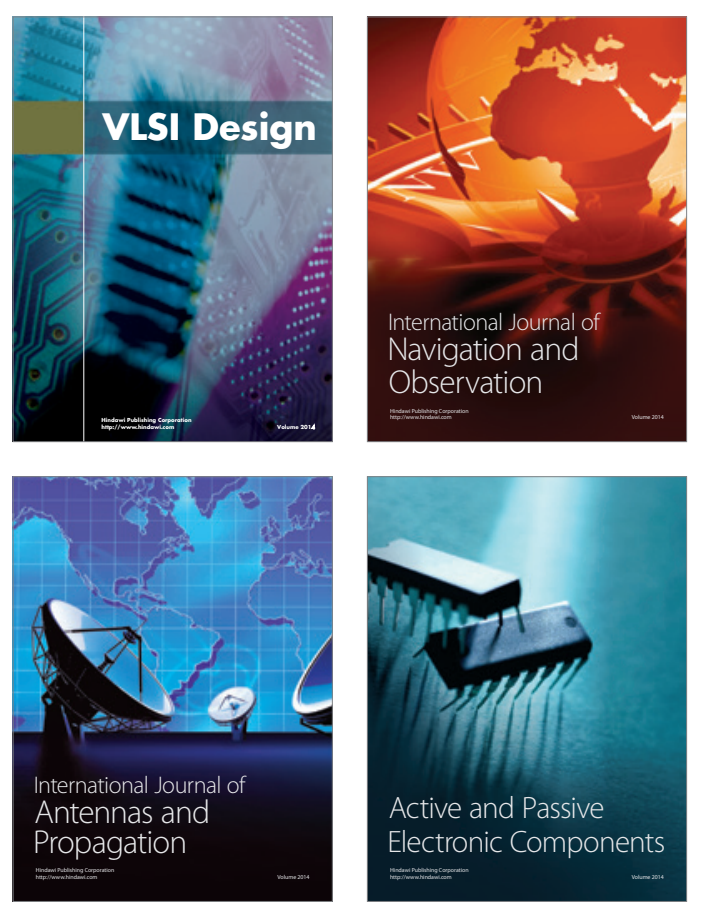
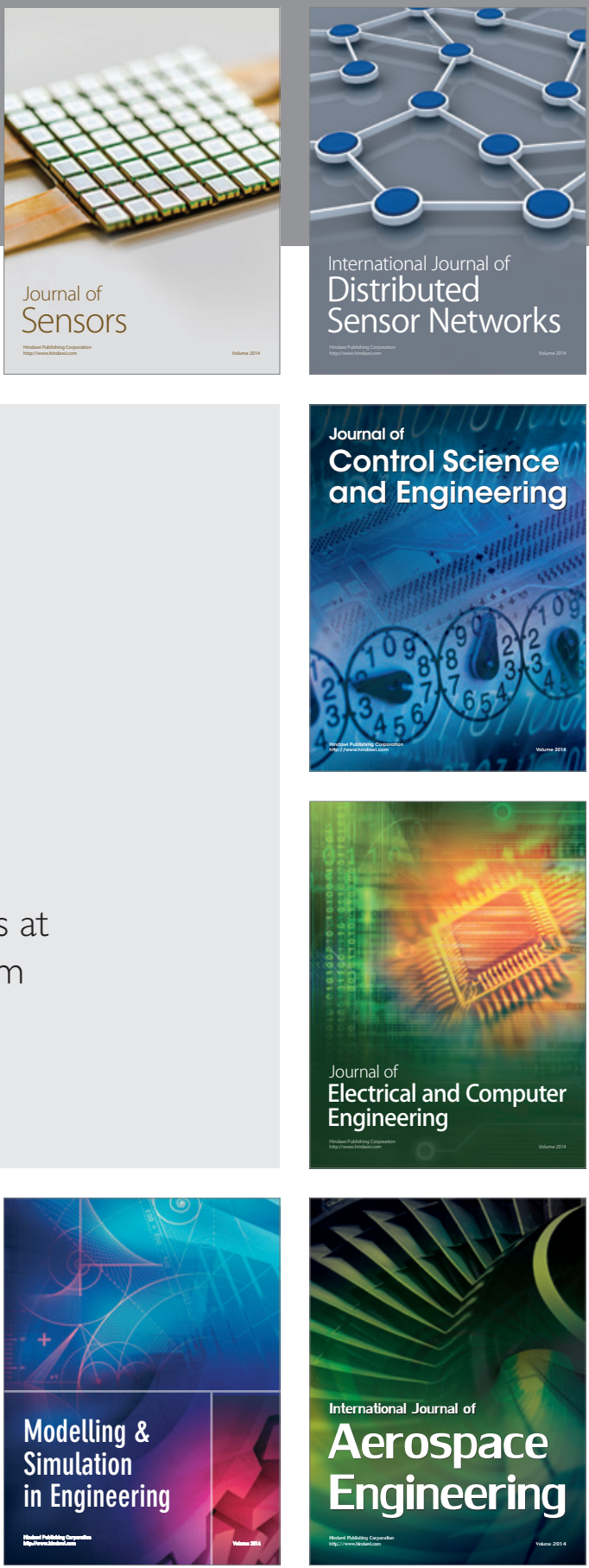

Journal of

Control Science

and Engineering
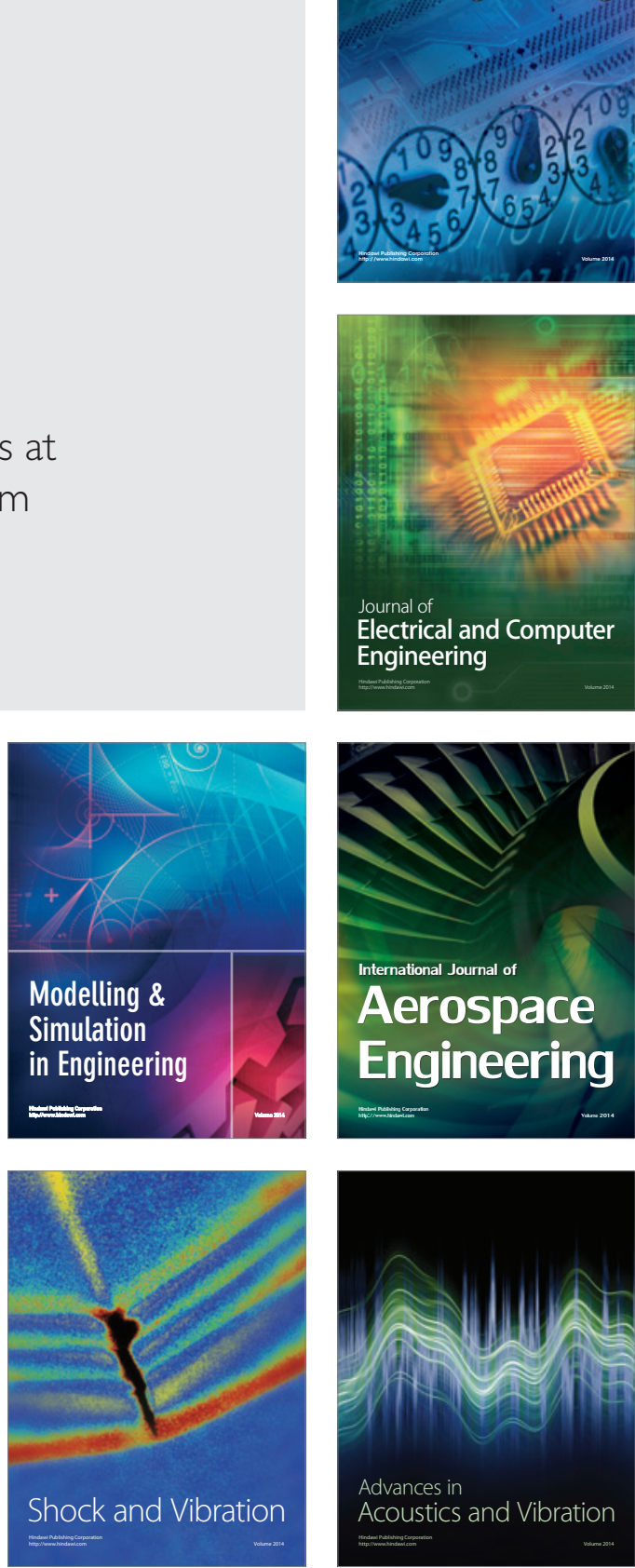